\title{
INTERPRETING TAMIL TEXT by USING MOBILE PHONE CAMERA
}

\author{
Jayasakthi velmurugan K. ${ }^{1}$, Dorairangaswamy M.A. ${ }^{2}$ \\ ${ }^{1}$ Research Scholar, Sathyabama University, Chennai, India, \\ jaisakthi21@gmail.com \\ 2 Professor, St.Peter's University, Chennai, India, \\ drdorairs@yahoo.co.in
}

\begin{abstract}
Interpreting Tamil Text from Signboard is a well-known image processing technology. This Process consists of four steps: capture the image from mobile Phone camera, pre-processing, Text Area detection, Tamil Text extraction, character segmentation and character recognition. Signboard plates are available in various styles and colors in various Places. Every State has its own National Language Text. Each LPR system use different combination of algorithms. The information which is present in the signboard image can be detected by using the mobile phone camera. Once the Tamil Text is detected it will be converted into whatever the form which is understandable by the non native user.
\end{abstract}

Keywords - pre-processing, Text detection, Text extraction, segmentation, recognition.

\section{INTRODUCTION}

Signboard information plays an important role in our society. Their format is often concise and direct, and the information they give is usually very useful. However, the foreign visitors may not understand the language that the signboard is written in, with the consequently loss of all that important information. The gaining momentum of portable mobile devices, the growing of their computational power and the inclusion of cheap digital cameras on them makes it possible to change from the classical hand dictionary translation to a new faster, comfortable and affordable way. In this sense, it is expected that a high percentage of the world population will own a mobile phone with an embedded camera, which is all that our system needs.

We propose, then, a method that detects the text Information within an image taken with a mobile phone Camera, extracts it, recognizes it and translates and phonetically transcribes it from Tamil to English language. This method may have to deal with different such as: low resolution and low quality images, perspective distortion due to the viewing angle used when taking the picture, blurring effects due to object movement or incorrect camera focusing, uneven lighting from shadowing, reflections, or inter-reflections, and multiple text formats within the same image. Nevertheless, since the computational resources are limited, our system is based on non-expensive algorithms in order to achieve the highest accuracy in the lowest processing time possible.

\section{SYSTEM ARCHITECTURE}

The proposed system deals with Mobile Phone camera which is used to recognize the Tamil Text and the interpreted English Message is given back to the User. From that message the user may able to know the information which is existing in the signboard.

In the below Fig.1, the camera becomes acting as a front end of the mobile phone. Once the signboard image is taken by the camera, the image is given to the back end of the system. It will do the work of finding the text message and converting it into an English text which is understandable by the user. 


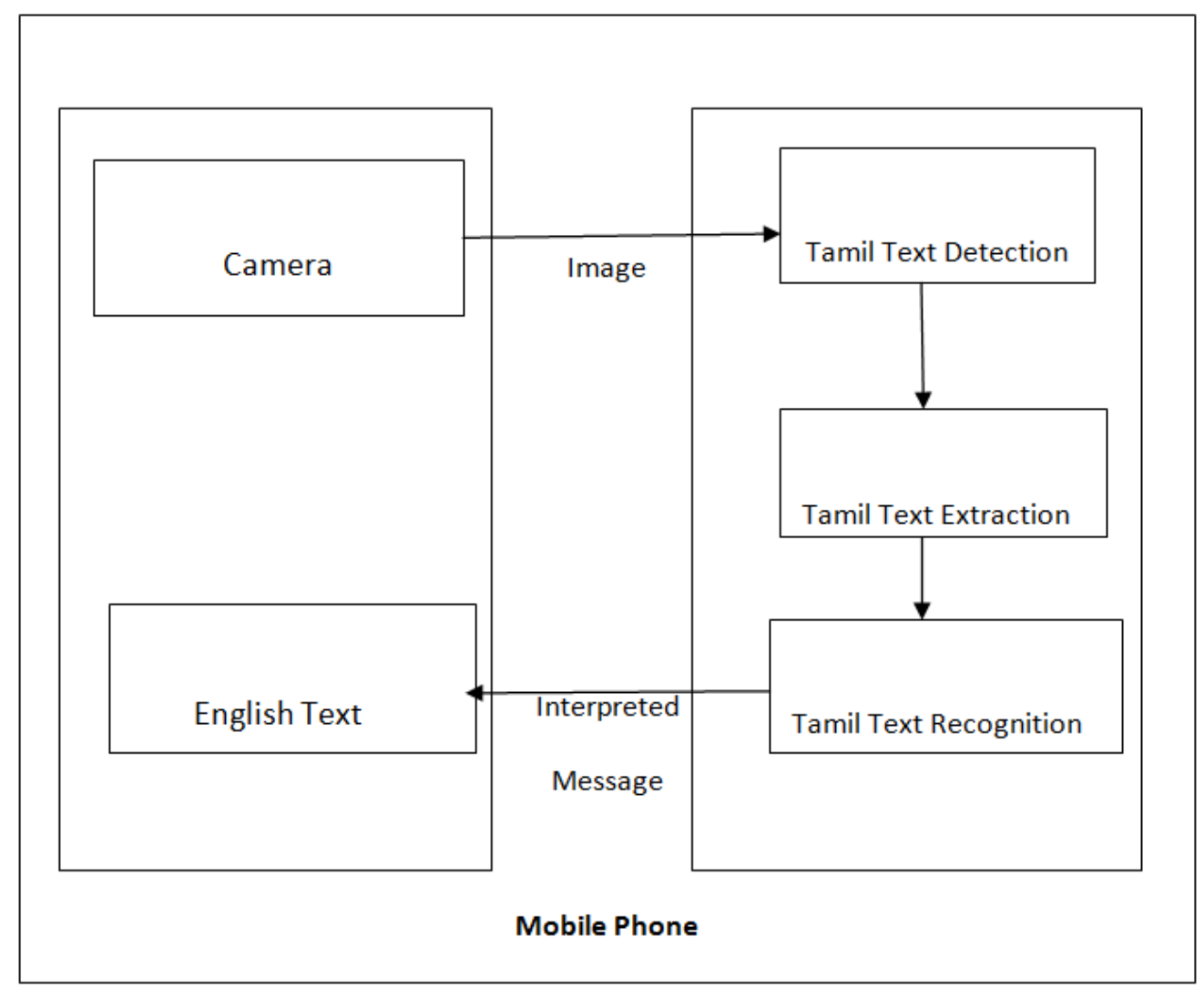

Fig. 1 System Architecture

\section{PROPOSED METHOD}

Proposed system is mainly based on Mobile Phone camera which is used for Tamil Text Message recognition followed interpreting it into an English Message. It deals with radial basis function which is discussed below.

\section{A. Radial Basis Function}

Radial Basis Function (RBF) is an approach of Neural Network (NN) which allows viewing a design as a curve fitting problem. The basic form of RBF NN comprises of three layers RBF NN is a mapping function which map from non-linearly separable space to linearly separable space. Due to these benefits RBF was used in this research not only for recognition but also for detection purposes

\section{B. Materials and Methods}

The proposed system is divided into four (4) main phases: The main phases are: Text Area detection, Tamil Text extraction, character segmentation and character recognition

1) Text Area Detection: The Text Area Detection is first stage is input to the Signboard image and the output is a portion of the image, and the Text Message normally has a rectangular shape, it can be extracted by identify all possible rectangle in the image which is shown in Fig. 1 and Fig. 2. It can used to the sobel filter technique this filter is used to detect the edge, The edge are two horizontal lines when performing horizontal edge detection, two vertical lines when performing vertical edge detection and a complete rectangle means when performing same times and proposed VEDA algorithm it is faster than Sobel filters by 7 (or) 9 times.

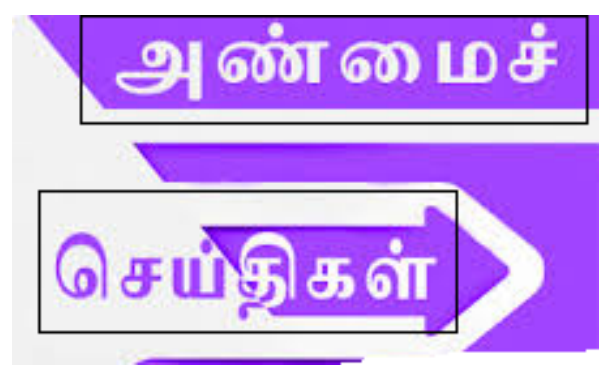

Fig. 2. Text Area detection 


\section{அ60் 60மச்}

\section{செய்திகள்}

Fig. 3. Vertical projection of Text Image

The maximum value of $p_{x}(x)$ corresponding to the axle of band can be computed as per Eq. 1:

$\mathrm{x}_{\mathrm{an}}=\arg \max \left\{\mathrm{p}_{\mathrm{x}}(\mathrm{x})\right\}$

The $\mathrm{X}_{a 0} y$ and $\mathrm{y}_{a 1} y$ are coordinates of band, which can be detected by Eq. 2:

$$
\begin{array}{ll}
\mathrm{x}_{\mathrm{a} 0}=\max \left\{\mathrm{x} \mid \mathrm{p}_{\mathrm{x}}(\mathrm{Y}) \leq \mathrm{c}_{\mathrm{x}} \cdot \mathrm{p}_{\mathrm{x}}\left(\mathrm{x}_{\mathrm{an})}\right\}\right. & \mathrm{x}_{0} \leq \mathrm{x} \leq \mathrm{x}_{\mathrm{an}} \\
\mathrm{x}_{\mathrm{a} 1}=\min \left\{\mathrm{x} \mid \mathrm{p}_{\mathrm{x}}(\mathrm{x}) \leq \mathrm{c}_{\mathrm{x}} \cdot \mathrm{p}_{\mathrm{x}}\left(\mathrm{x}_{\mathrm{bm})}\right\}\right. & \mathrm{Y}_{\mathrm{an}} \leq \mathrm{y} \leq \mathrm{y} 1
\end{array}
$$

Text Detection Algorithmic steps are,

let $\mathbf{L}$ to be a list of detected candidates

for $i:=0$ to number_of_bands_to_be_detected do

begin

detect $\mathrm{x}_{a 0}$ and $\mathrm{x}_{a 1}$ by analysis of projection $\mathrm{p}_{\mathrm{x}}$

save $\mathrm{a}_{0 \mathrm{y}}$ and $a_{1 y}$ to a list $\mathbf{L}$

zeroize interval $<\mathrm{x}_{\mathrm{a} 0}, \mathrm{X}_{\mathrm{a} 1}>$

end

2) Tamil Text Extraction: Tamil Text extraction is WT (Wavelet Transform) technique is using for the extracting the texture of the Message Plane. There are 2 sub bands HL is a first sub band HL denoted by vertical edge information and LH is denoted by Horizontal edge information. The maximum change in horizontal edge, the WT accuracy of $97.33 \%$. And proposed used technique is AB (Adaptive Boosting) is combined with Haarlike features to obtained Tamil Text extraction, the AB using a image of different format, size, and lighting condition, the method detection rate of $99 \%$.

Algorithmic Steps for Extraction

Step1: To convert into binary image.

Step2: To find how many white shapes are in the image (NP numbers).

Step3: To calculate and find the area of the numbers.

Step4: To store in one cell.

Step5: Compare with our database.

3) Character Recognition: The Tamil character recognition process deals with the character recognition. Although there are many techniques present and applied for character recognition like statistical, syntactic and neural networks in this research, character recognition is performed by using feature extraction. The first stage is Tamil Text extracted, another stage segmentation last stage is recognition, the previous stage is segmentation identify the character from image, all the character are identify is called recognition process.

\section{செய்திகள்}

Fig. 4. Character Recognition
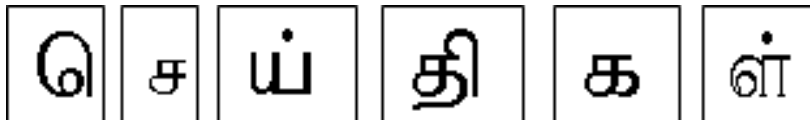

Fig.5. Character Segmentation

It Extract the character from the image using orientation algorithm which separates the character in terms of Horizontal and vertical wise. Boundary box is also used to remove the noise. 


\section{செய்திகள்}

Fig. 6. Before Segmentation

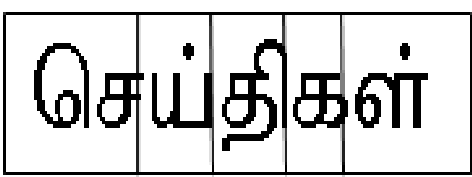

Fig. 7. After Segmentation

Thresholding is used to separate the character. To fix the thresholding value for one image. To read the each and every pixel to check the value of corresponding value of pixel and compare with that thresholding value. If thresholding value is less means that will be as put as 1 (white).Otherwise that is 0 (BLACK) which is shown in Eq. 3.

$$
\Sigma(i, j)= \begin{cases}1 & \text { if }(\mathrm{i}, \mathrm{j})>\text { threshold } . \\ 0 & \text { else }\end{cases}
$$

Seed-Fill Algorithm is used to extract the piece of information. The goal of the piece extraction algorithm is to find and extract pieces from a segment of the plate.

Step1: Let piece $\mathrm{R}$ be a set of (neighboring) pixels $[x, y]$

Step 2: Let $S$ be a set of all pieces R from a processed segment defined by the function $f(x, y)$.

Step 3: Let $X$ be a set of all black pixels: $X=\{[x, y] f(x, y)=1\}$

Step 4: Let $A$ be an auxiliary set of pixels

\section{IMPLEMENTATION AND RESULTS}

The Lenovo Mobile 6MP camera is used to operate the proposed system. By using the camera signboard image is taken and the Tamil text detection along with the maximum value in terms of percentage is shown in Fig. 7. It also uses some filters like median filter to get the clear image about the characters in number plate which is shown in Fig. 8.

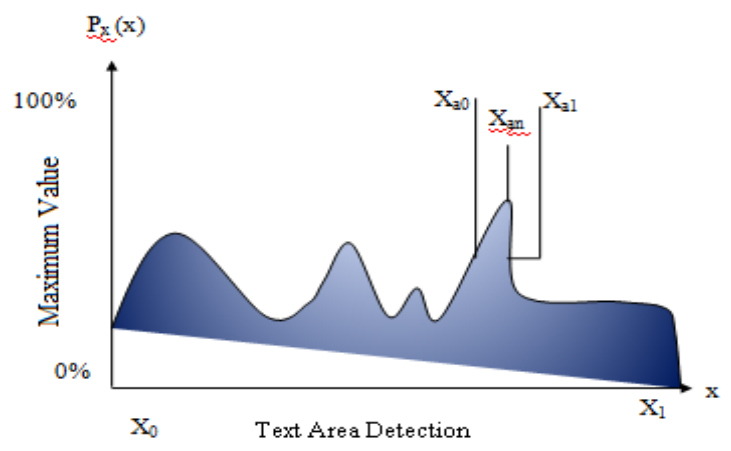

Fig. 8. Maximum value of Text Area Detection

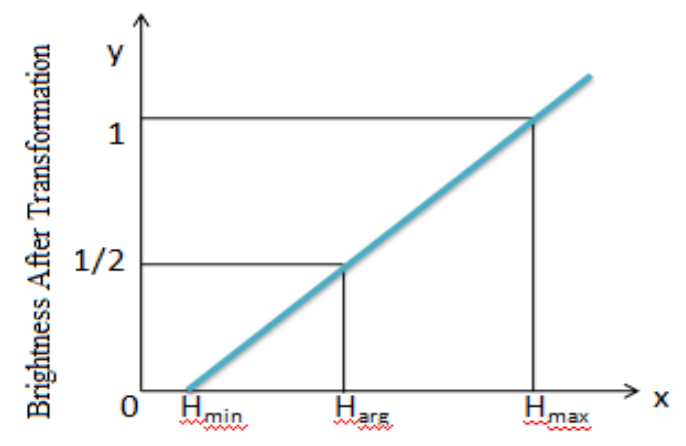

Brightness Before Transformation

Fig. 9. Median filter with contrast of characters 
The performance analysis of proposed system is tested using MATLAB simulator which is summarized in Table. 1.

TABLE I. PERFORMANCE ANALYSIS of TTR SYSTEM

\begin{tabular}{|l|c|}
\hline \multicolumn{1}{|c|}{ Criteria } & TTD \\
\hline Number of Signboard images Tested & 10 \\
\hline Estimated Speed & $62.3[\mathrm{~km} / \mathrm{hr}]$ \\
\hline Simulation Tool & MATLAB \\
\hline Simulation Time & 15 mins \\
\hline
\end{tabular}

The research design is divided into four (4) main phases: The main phases of a TTR process are: License Plate Detection, Extraction, Character Recognition and Character Segmentation. The phases of variation under different style of Tamil Text images are shown in Fig. 9.

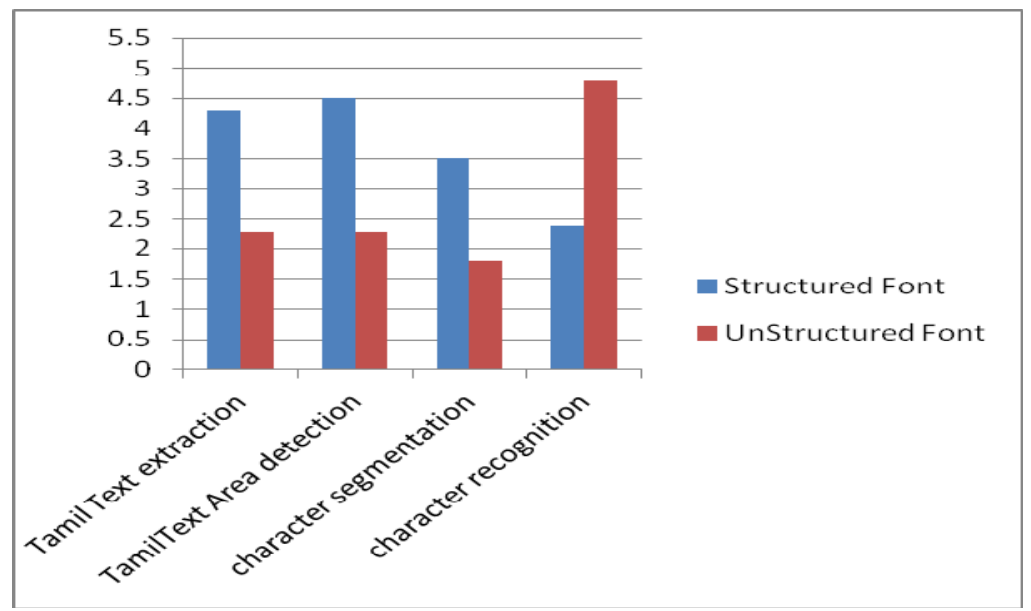

Fig. 10. Different Style of Tamil Text Images

\section{V.CONCLUSION}

We proposed a system to translate signboard images taken with a mobile phone camera from Tamil to English. Since the computational resources of these devices are limited, we had to use fast, simple and accurate possible algorithms to work in the most common situations.

\section{REFERENCES}

[1] Adrian Canedo-Rodríguez,Soohyung Kim,Jung H. Kim,Yolanda Blanco-Fernández "English to Spanish Translation of Signboard Images from Mobile Phone Camera”, IEEE Conferences on SOUTHEASTCON'09,May 2009.

[2] Yu Zhong, Hongjiang Zhang and Jain A.K, "Automatic caption localization in compressed video," IEEE Transactions on Pattern Analysis and Machine Intelligence, Volume 22, Issue 4,April, 2000, pp. 385-392.

[3] Canny, J., A Computational Approach To Edge Detection, IEEE Trans. Pattern Analysis and Machine Intelligence, 8:679-714, 1986

[4] Xilin Chen, Member, IEEE, Jie Yang, Member, IEEE, Jing Zhang, and Alex Waibel, “Automatic Detection and Recognition of Signs From Natural Scenes". IEEE Transactions on Image Processing, Vol. 3, No.1, January 2004.

[5] Keechul Jung, Kwang In Kim, Anil K. Jain, "Text Information Extraction in Images and Video: a Survey", Pattern Recognition, 37(5):977-997, 2004.

[6] J. Ohya, A. Shio, and S. Akamastsu, TMRecognizing Characters in Scene Images, IEEE Trans. Pattern Analysis and Machine Intelligence, vol. 16, pp. 214 $220,1994$.

[7] B. Shen and I.K. Sethi, ${ }^{\text {TM} C o n v o l u t i o n-B a s e d ~ E d g e-D e t e c t i o n ~ f o r ~ I m a g e / V i d e o ~ i n ~ B l o c k ~ D C T ~ D o m a i n, ~}{ }^{\circ}$ J. Visual Comm. and Image Representation, vol. 7, no. 4, pp. 411 $\pm 423,1996$.

[8] Gao, J. and Yang, J., "An Adaptive Algorithm for Text Detection from Natural Scenes," Proceedings of Computer Vision and Pattem Recognition (CVPR 2001).

\section{AUTHOR PROFILE}

K.Jayasakthi velmurugan is doing Research in sathyabama university. It is located in Chennai, Tamilnadu, India.

Dr.M.A.Dorairangaswamy is a Professor and working in St.Peter's University. It is located in Chennai,Tamilnadu,India. 